\title{
The electromagnetic signature of jets
}

\author{
Charles Gale†, T. C. Awes§, Rainer J. Friesథand Dinesh K. \\ Srivastava \\ $\dagger$ Department of Physics, McGill University, 3600 University Street, Montreal, \\ QC, H3A 2T8, Canada \\ $\S$ Oak Ridge National Laboratory, Oak Ridge, Tennessee 37831, USA \\ I School of Physics \& Astronomy, University of Minnesota, 16 Church Street \\ S.E., Minneapolis, Minnesota 55455, USA \\ ‡ Variable Energy Cyclotron Centre, 1/AF Bidhan Nagar, Kolkata 700 064, \\ India
}

\begin{abstract}
.
Recent RHIC data have suggested an interesting scenario where jets, after being formed in the very first instants of the nuclear collision, interact strongly and are absorbed by the hot and dense matter subsequently created. In this respect, electromagnetic signals constitute another class of penetrating radiation. We first propose the study of large mass dileptons induced by the passage of highenergy quark jets through quark-gluon plasma. We find that the yield due to the jet-plasma interaction gets progressively larger as the collision energy increases: it is slightly less than the Drell-Yan contribution at RHIC energies, and it is a factor of 10 larger than Drell-Yan at the LHC. In a related investigation, we then propose to study dilepton-tagged jets. We estimate quantitatively the various background sources, and identify windows where those are sub-dominant.
\end{abstract}

\section{Introduction}

Relativistic heavy ion collisions currently constitute a paradigm for studies of matter under extreme conditions. In a complex many-body environment whose existence spans a wide range of dynamical variables, several complementary observables are needed to identify new physics. Hard hadronic probes, as measured by the current generation of RHIC experiments, seem to support a very interesting scenario where the QCD jets being formed in the first instants of the collision are being quenched by the hot and dense matter [1]. Albeit compelling, the experimental evidence still needs to be interpreted in a quantitative and systematic theory in order to clearly distinguish effects in the final state from effects in the initial state, for example [2]. Jet-quenching is in fact a strong jet-plasma interaction. If such phenomena occur, the same interactions should yield other products. Recalling the need to identify new phenomena with different measurements, we have calculated the production of electromagnetic radiation resulting from the passage of jets through a quark-gluon plasma. An observation of this new source would confirm the occurrence of the conditions suitable for jet-quenching to take place. 


\section{Formulation and results}

We propose that the phase space distribution of partons in the hot and dense medium can be decomposed as

$$
f(\boldsymbol{p})=f_{\mathrm{th}}(\boldsymbol{p})+f_{\mathrm{jet}}(\boldsymbol{p}),
$$

where the plasma component is approximated by a thermal distribution with a temperature $T: f_{\operatorname{th}}(\boldsymbol{p})=\exp (-E / T)$. The jet component is calculated from perturbative QCD, and is limited to a region $p_{\mathrm{T}} \gg 1 \mathrm{GeV}$. This separation is tenable kinematically, as jet spectra fall of as a power law and can thus be differentiated from the thermal component. The phase space distribution for jets can be obtained from perturbative QCD calculations, with an appropriate normalization allowing for the space-time extent of the emitting source [3]. Given the parton distribution functions, one may calculate the production rate for real photons from jet-plasma interactions, as well as those for lepton pairs. For real photons, this exercise has been carried out recently [4]. The details of this calculation will not be discussed here, but they are similar to those in the virtual photon case, considered now. It suffices here to say that the new photon source is dominant, up to $p_{\mathrm{T}} \approx 6 \mathrm{GeV} / \mathrm{c}$. Since lepton pair spectra offer the added flexibility of having two independent variables (invariant mass and three-momentum, for example), it is imperative to investigate the effect of the new production mechanism.

We consider lepton pairs produced by quark-antiquark annihilation, where one parton is thermal and the other is a hard QCD jet. The obvious background to our new signal will be that from Drell-Yan processes, which we also compute. We have estimated the jet and Drell-Yan production in lowest order pQCD, using CTEQ5L parton distributions and EKS98 shadowing corrections [5]. As a first step, a thermally and chemically equilibrated plasma is assumed to be created at proper time $\tau_{0}$. An isentropic expansion will yield the usual correlation between the measured charged particle multiplicity and initial temperature [6]. The details are to be found in [5]. One obtains the result shown in Fig. 1 for symmetric collisions of lead nuclei at RHIC energies. The two curves for the thermal and jet-plasma interactions are associated with $\tau_{0}=0.15$ and $0.5 \mathrm{fm} / \mathrm{c}$, top and bottom respectively. The higher temperature (shorter formation time) represents a rapid thermalization limit set by the uncertainty principle [7]. We have explored the sensitivity of our results to this assumption, by looking also at perhaps more conservative initial conditions. In fact, we see that the new signal is relatively robust with respect to temperature variations, as it only depends linearly on the thermal distributions. This is not the case for the purely thermal radiation, a "classic" plasma signature. At RHIC, jet-plasma interactions produce more virtual photons than the annihilation of thermal partons, but less than the Drell-Yan mechanism. However, the results at LHC energies, Fig. 2] show that the larger initial temperatures lead to an excess of large mass dileptons over those from conventional sources. Note that the usual contribution from correlated heavy quark decays is not shown here.

Another aspect of jets and electromagnetic radiation we wish to highlight is the following. At the parton level, the processes $q g \rightarrow q \gamma^{*}$ and $q \bar{q} \rightarrow g \gamma^{*}$, are next-toleading order (in $\alpha_{s}$ ), however those are very effective in producing large momentum lepton pairs. These will be correlated with a recoiling parton which will traverse and, in principle, interact with an eventual plasma. The jets will thus have a dilepton tag which, combined with a jet reconstruction measurement, could yield valuable 


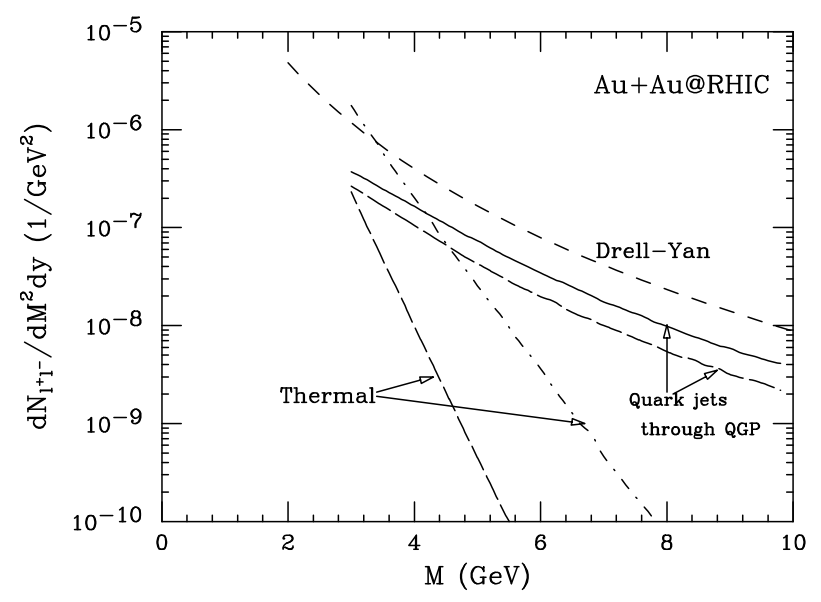

Figure 1. Dilepton spectrum from central $\mathrm{Pb}+\mathrm{Pb}$ collisions at $\sqrt{s_{\mathrm{nn}}}=200$ $\mathrm{GeV}$. The contributions from Drell-Yan, thermal, and jet-plasma interactions are shown. More details in the text and references.

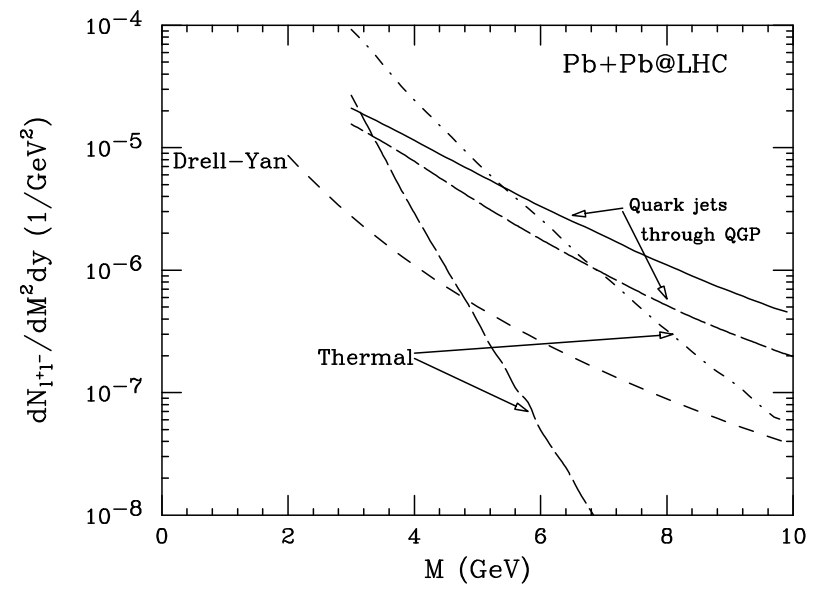

Figure 2. Same as Fig. 11 for $\mathrm{Pb}+\mathrm{Pb}$ collisions at $\sqrt{s_{\mathrm{nn}}}=5.5 \mathrm{TeV}$.

information about in-medium energy modification. Photon-tagged jets have been suggested as a probe for jet-quenching studies [8], but we argue that virtual photons offer some advantages [9]. This last statement is illustrated in Fig. 3] where the lepton pair signal (DY@NLO) is compared to the known background from correlated heavy quark decays. Clearly, there exists a window in invariant mass and transverse momentum where the electromagnetic signal from the jet tag outshines that from the background. This assertion holds true at RHIC (Fig. 3) and, to a somewhat lesser extent, at the LHC [9]. 


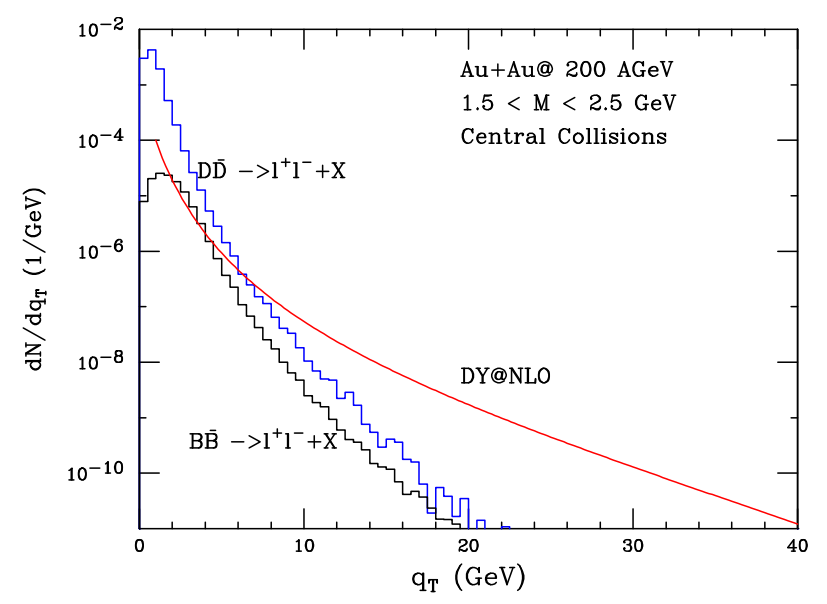

Figure 3. Dilepton signal associated with a recoiling jet compared with spectra from correlated heavy quark ( $c$ and $b$ ) decays, at RHIC energies.

\section{Summary}

Jet-plasma interactions provide a new source of virtual photons that can compete with the Drell-Yan process and even outshine it, depending on the energy. In a somewhat more utilitarian vein, dileptons can also be used to tag jets and thus provide valuable information on their characteristics at formation time.

\section{Acknowledgments}

C.G. is supported in part by the Natural Sciences and Engineering Research Council of Canada, and in part by the Fonds Nature et Technologies of Quebec. ORNL is managed by UT-Battelle, LLC, for the U.S. Department of Energy under contract DE-AC05-00OR22725. R.J.F. is supported by DOE grant DE-FG02-87ER40328.

\section{References}

[1] Vitev I these proceedings

[2] Jalilian-Marian J these proceedings

[3] Lin Z and Gyulassy M 1995 Phys. Rev. C 512177

[4] Fries R J, Müller B and Srivastava D K 2003 Phys. Rev. Lett. 90132301

[5] Srivastava D K, Gale C and Fries R J 2003 Phys. Rev. C 67034903

[6] Bjorken J D 1983 Phys. Rev. D 27 140; Hwa R C and Kajantie K 1985 Phys. Rev. D 321109

[7] Kapusta J, McLerran L D and Srivastava D K 1992 Phys. Lett. B 283145

[8] Wang X N, Huang Z and Sarcevic I 1996 Phys. Rev. Lett. 77 231; Wang X N and Huang Z 1997 Phys. Rev. C 553047

[9] Srivastava D K, Gale C and Awes T C 2003 Phys. Rev. C 67054904 